\title{
In situ analysis of apoptosis in Aspergillus nidulans with ethidium bromide and acridine orange
}

\author{
S.M. Leles, R. Factori and C.L.M.S.C. Rocha \\ Departamento de Biotecnologia, Genética e Biologia Celular, \\ Universidade Estadual de Maringá, Maringá, PR, Brasil \\ Corresponding author: C.L.M.S.C. Rocha \\ E-mail: clmscrocha@yahoo.com.br
}

Genet. Mol. Res. 12 (3): 2895-2901 (2013)

Received June 14, 2012

Accepted September 24, 2012

Published August 12, 2013

DOI http://dx.doi.org/10.4238/2013.August.12.5

\begin{abstract}
Apoptosis and necrosis are among several types of cell death. We stained the nuclei of Aspergillus nidulans grown in microcolonies with ethidium bromide and acridine orange to detect in situ apoptosis. Suspensions of conidia from 5-day-old colonies of the $A$. nidulans strains biA1methG1, G422, CLC100, and CLB3 were each put into two tubes. The suspension of one tube was irradiated with ultraviolet light for $20 \mathrm{~s}$, whereas the other tube was not exposed to irradiation. The two suspensions were inoculated in complete liquid medium and $50-\mu \mathrm{L}$ samples were placed on sterilized cover slips, spread on the surface of solid culture media on Petri dishes. After the micro-colonies were formed, the material on the cover slips was stained with ethidium bromide and acridine orange, placed on the lamina and observed under a fluorescence microscope. This staining method was efficient in discriminating normal nuclei from those going apoptosis and necrosis. Results have shown that irradiation provokes apoptosis but does not induce necrosis. There were no differences between the three strains and all data were considered to be statistically significant.
\end{abstract}

Key words: Staining of nuclei; Fluorescent stains; Detection of apoptosis 


\section{INTRODUCTION}

Apoptosis is a process of programmed cell death ruled by numberless genes and phylogenetically preserved during the evolution. Its analysis is thus possible in Aspergillus nidulans, an eukaryotic model used in developmental genetics. In 2002, Sydney Brenner, John Sulston, and Robert Horvitz were awarded the Nobel Prize for the identification of genes that regulate apoptosis in Caenorhabditis elegans. Henceforth, interest in revealing and controlling the activation and blocking mechanisms of the apoptotic process increased for applications in degenerative diseases such as Alzheimer's and cancer (Kerr et al., 1972; Majno and Joris, 1995; Solary et al., 1996; Metzstein et al., 1998; Jeon et al., 2002; Amarante-Mendes, 2003; Hamann et al., 2008).

Several factors, such as the welding of molecules to membrane receptors, chemical and therapeutic agents, ionizing radiation, DNA damage, and high levels of reactive oxygen species, may trigger the onset of apoptosis. The main characteristics of programmed cell death are chromatin condensation, DNA fragmentation, and the formation of apoptotic bodies that may be absorbed by neighboring cells without causing any damage (Anazetti and Melo, 2007; Stevens et al., 2007).

Under normal conditions, apoptosis is indispensable for the development and maintenance of tissues and control of organ size because it is a process that maintains morphogenesis and immunity. Cell removal with genomic instability, which triggers homeostasis in organisms or, more accurately, the equilibrium between cell proliferation and cell death, also relies on apoptosis (Kerr et al., 1972; Cohen et al., 1992; Anazetti and Melo, 2007; Stevens et al., 2007).

Many methods to detect in vitro apoptosis are available, including ladder patterns in DNA electrophoresis (Kerr et al., 1972), terminal deoxyribonucleotidyl transferase-mediated dUTP-digoxigenin nick end labeling (Gavrieli et al., 1992), ethidium bromide (EB) and acridine orange (AO) (Cohen et al., 1992), Hoechst stain (Belloc et al., 1994), 4',6-diamidino-2-phenylindole (Tamm et al., 1998), caspases 3-7 activity (Tamm et al., 1998), and annexin V (Hammill et al., 1999).

Each method has its limitations, from multi-stage procedures to time-consuming assays and the possibility of damaging cell membranes. Another common difficulty in many tests is the simultaneous counting of normal, apoptotic, and necrotic cells. The occurrence of a false (apoptotic) positive reaction is possible and would engage the researcher in a further morphological analysis (Renvoize, 1998).

Staining with fluorescent AO and EB to detect apoptosis is a technique used in several cell types. This staining simultaneously quantifies and differentiates all types of normal cells in either apoptosis or necrosis. It is a simple, fast, and precise method for apoptosis classification (McGahon et al., 1995; Ribble et al., 2005; Tritz et al., 2008). This study standardized the $\mathrm{EB}$ and $\mathrm{AO}$ nuclear staining technique to detect apoptosis in micro-colonies of the ascomycete A. (= Emericella) nidulans under normal conditions, and conditions of genetic damage caused by exposure to ultraviolet light.

\section{MATERIAL AND METHODS}

Strains biA1methG1 and G422 from the University of Glasgow (Scotland) were used in the current study. Strains CLB3 and CLC100 were also used and obtained from the Laboratory of Molecular Genetics and Development of Universidade Estadual de Maringá. Conidia from all strains were collected from 5-day-old growing colonies in complete solid medium at $37^{\circ} \mathrm{C}$ (Pontecorvo et al., 1953; Clutterbuck, 1974), and placed in a water solution with $0.01 \%$ 
(v/v) Tween 80. The suspension was filtered in sterilized glass wool after heavy shaking. Each suspension was placed in 2 test tubes - control and ultraviolet - with complete liquid medium (Pontecorvo et al., 1953; Clutterbuck, 1974). The suspension in the control tube received no irradiation; the suspension in the ultraviolet tube was irradiated for $20 \mathrm{~s}$ with ultraviolet $\mathrm{B}$ (UVB) radiation $\left(0.24 \mathrm{~mJ} / \mathrm{cm}^{2}\right)$.

After the cultures were prepared, $50-\mu \mathrm{L}$ samples from each suspension were inoculated on sterilized cover slips and placed on the surface of solid medium on Petri dishes. The plates were incubated at $37^{\circ} \mathrm{C}$ for 8 and $24 \mathrm{~h}$. Micro-colonies were then stained on the cover slip with $10 \mu \mathrm{L}$ stain in a $1: 1$ proportion of $100 \mu \mathrm{g} / \mathrm{mL}$ AO and $100 \mu \mathrm{g} / \mathrm{mL}$ EB, and immediately observed under an AXIOSKOP 2 PLUS fluorescence microscope (Zeiss, Berlin, Germany) with fluorescein isothiocyanate and Rhodamine filters at 100X magnification. Images were captured with a digital camera and evaluated using Axion Vision 4.8 (Zeiss). Images were retrieved and underwent counting for 200 nuclei. Analysis of variance and the Fisher least significant difference test compared results among averages. All analyses were performed using Statistica 7.1 (Statsoft Inc., 2005)

\section{RESULTS}

Although the possibility existed that the wall of the hyphae would be thick enough to make stain absorption difficult, stains were absorbed by the hyphae and stained the nuclei. Another expected difficulty was that the 24-h hyphae would be less stained than those at $8 \mathrm{~h}$. However, nuclei staining occurred regardless the developmental stage of the hyphae ( 8 or $24 \mathrm{~h}$ ).

Stain absorption by nuclei in the hyphae was classified normal, apoptotic, and necrotic based on the morphological differences among the nuclei under different conditions. Figure 1A shows normal green-stained integral nuclei with normal diameters. Figure 1B shows that necrotic nuclei were red-orange-stained integral nuclei with a normal diameter. Figure 1C shows nuclei at initial apoptosis, green-stained nuclei with condensed chromatin and, thus, a smaller diameter. Figure 1D shows nuclei in final apoptosis, red-orange-stained nuclei with highly condensed and fragmented chromatin that forms apoptotic bodies.

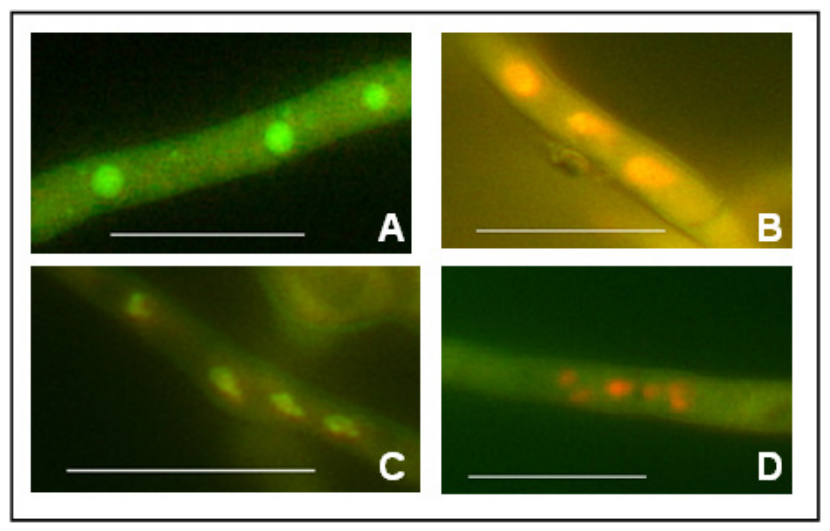

Figure 1. Morphological aspect of hypha nuclei of micro-colonies, with $24 \mathrm{~h}$ in a complete liquid medium at $37^{\circ} \mathrm{C}$, stained with acridine orange and ethidium bromide. Scale bar $=10 \mu \mathrm{m}$. A. Normal nuclei; B. necrotic nuclei; C. nuclei at the start of apoptosis; D. nuclei at the end of apoptosis. 
Each strain had a standard in the control, as expected (Figure 2), although all strains showed that the average of the normal nuclei was significantly higher than that of apoptotic nuclei, which in its turn, was higher than that of necrotic nuclei. However, the number of apoptotic nuclei in the UVB-treated conidia was significantly higher than that in normal nuclei.
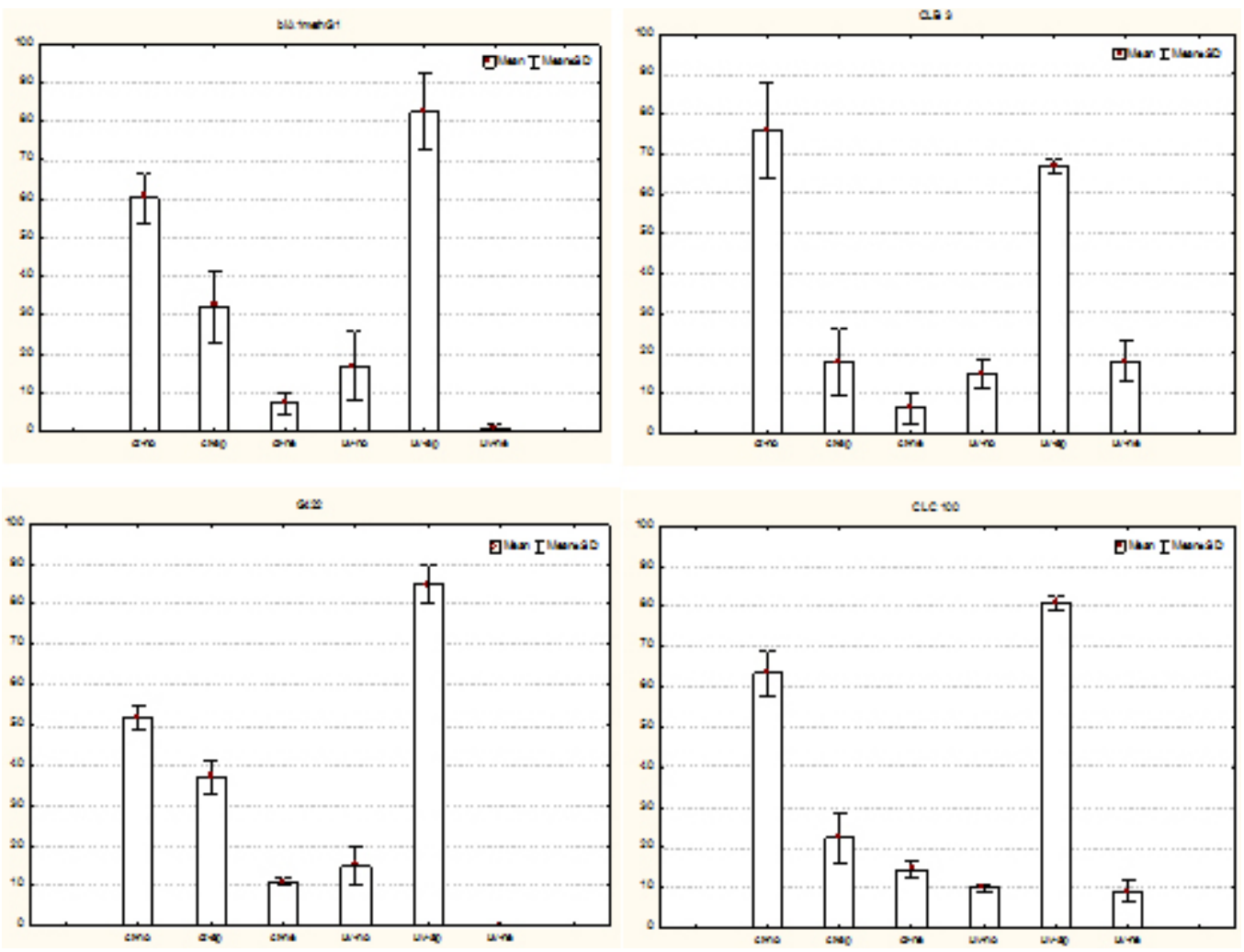

Figure 2. Statistical analysis of nucleus conditions (normal, apoptotic, and necrotic) in strains at control and ultraviolet light-treated conditions. cl-no $=$ control condition, normal nuclei; cl-ap = control condition, apoptotic nuclei; cl-ne $=$ control condition, necrotic nuclei; uv-no = ultraviolet condition, normal nuclei; uv-ap = ultraviolet condition, apoptotic nuclei; uv-ne = ultraviolet condition, necrotic nuclei. $\mathrm{SD}=$ standard deviation.

Statistical analyses of the results demonstrated no difference between the average number of necrotic nuclei in the controls and that in the UVB-treated nuclei. This result showed that UVB did not trigger necrosis. nidulans.

The examination of stained nuclei confirmed the pro-apoptotic activity of UVB in $A$.

\section{DISCUSSION}

Current assays adapt the nuclear staining technique described by Doonan and Cotter (2008) when the morphological study of apoptosis is possible. 
Fluorescent staining intercalated with DNA is used for the characterization. AO penetrates healthy cells, stains the nuclei green, and reveals organized chromatin. Cells in early apoptosis have a condensed nucleus with highly condensed green-stained chromatin. EB, captured only by cells that have lost integrity in their cytoplasmic membrane, stains cells orange-red. Late apoptotic cells have orange-red-stained nuclei and extremely condensed or fragmented chromatin. Nuclei in the necrosis stage are stained orange-red with organized chromatin (McGahon et al., 1995; Coligan et al., 1995; Ribble et al., 2005).

Wyllie (1980) has described a technique for apoptosis detection using DNA electrophoresis. The method has been used to visualize apoptosis in various cell types submitted to different unleashing situations (Arnoult et al., 2002). However, the method makes use of DNA isolation of cells and the observation of its fragmentation compared with ladder standard that characterizes the DNA of apoptotic cells.

Recently, developed staining techniques of apoptotic nuclei have triggered in situ analyses of various tissues and organisms and, consequently, increased the resources available for the study of the mechanism of apoptosis (Telford et al., 1992; Jeon et al., 2002; Tritz et al., 2008).

The DNA intercalators EB and AO have been used in various assays to identify and quantify apoptotic cells. Their use has several advantages, such as rapidity and capability of simultaneously identifying all possible nuclear stages (Telford et al., 1992; Jeon et al., 2002; Ribble et al., 2005; Tritz et al., 2008; Dogan et al., 2011). Whereas AO stains normal nuclei (Rosenberger and Kessel, 1967), EB stains only cells that have lost membrane integrity (Renvoize et al., 1998).

Staining for apoptosis has already been used in cell culture (Dogan et al., 2011), histological cuts (Gavrieli et al., 1992), and fungi with cell walls. Protoplasts are obtained by the removal of the wall before staining (Leiter et al., 2005).

An important contribution of current technique is its application in the staining of nuclei in the integral micro-colonies of fungi. To undertake in situ analysis of mycelium in different phases of development, we used cultures on cover slips with liquid culture medium. The method facilitated handling and staining and thus the observation of the entire colony.

During the assay, we observed that the cell wall did not interfere with staining, even when EB was used. In other cell types, EB could penetrate cells with a destabilized membrane at the start of apoptosis (Kroemer et al., 2009). This fact seems irrelevant in A. nidulans because both AO and EB penetrated the cell wall of the hyphae and stained normal, apoptotic, and necrotic nuclei.

The use of a filamentous fungus proved to be viable for the analysis of apoptosis in situ. In contrast to other eukaryotes, this fungus has cytoplasm compartments with 2 to 10 nuclei that communicate via septal pores and form hyphae lined with a cell wall. The cell structure makes the concomitant analysis of several nuclei possible (Morris, 1975; Kempken and Kuck, 1996).

Various assays of each cell type have established specific times at which apoptosis can be observed (Arnoult et al., 2002; Tritz et al., 2008). The current results show that nuclei can be stained using the EB/AO method after $8 \mathrm{~h}$ of growth. However, staining for $24 \mathrm{~h}$ provided more information, because after this period, the conidia had formed micro-colonies and had a greater number of nuclei. The distribution of nuclei among the septa and in the conidiophore structures could be seen.

The great number of nuclei at the start of apoptosis, even in controls, is notorious in 
the current study. It has been mentioned by Hammill et al. (1999) related to their use of annexin to stain B cells of mice lymphoma. These authors have reported that many nuclei identified as apoptotic reverted and did not die. A different apoptosis situation was registered. The same researchers have suggested that cells stained as early apoptotic cells may be at a senescence stage, a type of permanent arrest of the cell cycle first described by Hayflick and Moorhead in 1961. Consequently, the nuclei may be registered as a type of "live-dead cell" that, in certain cases, may emerge from such a condition and complete the cycle (Hammill et al., 1999).

Observing this new type of cell condition featuring permanent arrest by means of the $\mathrm{AO} / \mathrm{EB}$ staining technique is an important methodological contribution to the study of the cell cycle and associated mechanisms (control of G1/S checking, repair, apoptosis).

The methodology standardized in the current analysis may be used as an alternative for the in situ analysis of apoptosis in micro-colonies of $A$. nidulans. It contributed to the study of the cell cycle owing to its efficiency in simultaneously identifying the normal, apoptotic, and necrotic nuclear stages. Furthermore, the simplicity and speed of the procedure maintain the integrity of the material analyzed, an essential element in the investigation of cell cycle mechanisms associated with tissue development.

\section{ACKNOWLEDGMENTS}

Research supported by Coordenação de Aperfeiçoamento de Pessoal de Nível Superior and Microscopy Central of Complexo de Centrais de Apoio à Pesquisa da Universidade Estadual de Maringá, for microscopy analysis.

\section{REFERENCES}

Amarante-Mendes GP (2003). Apoptose: programa molecular de morte celular. Einstein 1: 15-18.

Anazetti MC and Melo PS (2007). Morte celular por apoptose: uma visão bioquímica e molecular. Metrocamp Pesq. 1: $37-58$.

Arnoult D, Akarid K, Grodet A, Petit PX, et al. (2002). On the evolution of programmed cell death: apoptosis of the unicellular eukaryote Leishmania major involves cysteine proteinase activation and mitochondrion permeabilization. Cell Death Differ. 9: 65-81.

Belloc F, Dumain P, Boisseau MR, Jalloustre C, et al. (1994). A flow cytometric method using Hoechst 33342 and propidium iodide for simultaneous cell cycle analysis and apoptosis determination in unfixed cells. Cytometry 17: 59-65.

Clutterbuck AJ (1974). Aspergillus nidulans. In: Handbook of Genetics (King RC, ed.). Plenum Publishing, New York, 447-510

Cohen GM, Sun XM, Snowden RT, Dinsdale D, et al. (1992). Key morphological features of apoptosis may occur in the absence of internucleosomal DNA fragmentation. Biochem. J. 286 (Pt 2): 331-334.

Coligan JE, Kruisbeek AM, Margulies DH, Shevach EM, et al (1995). Current Protocols in Immunology. In: Related Isolation Procedures and Functional Assays (Coico R, ed.). Vol. 1. John Wiley \& Sons Inc., New York, 3.17.1.

Dogan I, Cumaoglu A, Aricioglu A and Ekmekci A (2011). Inhibition of ErbB2 by herceptin reduces viability and survival, induces apoptosis and oxidative stress in Calu-3 cell line. Mol. Cell Biochem. 347: 41-51.

Doonan F and Cotter TG (2008). Morphological assessment of apoptosis. Methods 44: 200-204.

Gavrieli Y, Sherman Y and Ben-Sasson SA (1992). Identification of programmed cell death in situ via specific labeling of nuclear DNA fragmentation. J. Cell Biol. 119: 493-501.

Hamann A, Brust D and Osiewacz HD (2008). Apoptosis pathways in fungal growth, development and ageing. Trends Microbiol. 16: 276-283.

Hammill AK, Uhr JW and Scheuermann RH (1999). Annexin V staining due to loss of membrane asymmetry can be reversible and precede commitment to apoptotic death. Exp. Cell Res. 251: 16-21.

Hayflick L and Moorhead PS (1961). The serial cultivation of human diploid cell strains. Exp. Cell Res. 25: 585-621. 
Jeon BW, Kim KT, Chang SI and Kim HY (2002). Phosphoinositide 3-OH kinase/protein kinase B inhibits apoptotic cell death induced by reactive oxygen species in Saccharomyces cerevisiae. J. Biochem. 131: 693-699.

Kempken F and Kuck U (1996). restless, an active Ac-like transposon from the fungus Tolypocladium inflatum: structure, expression, and alternative RNA splicing. Mol. Cell Biol. 16: 6563-6572.

Kerr JF, Wyllie AH and Currie AR (1972). Apoptosis: a basic biological phenomenon with wide-ranging implications in tissue kinetics. Br. J. Cancer 26: 239-257.

Kroemer G, Galluzzi L, Vandenabeele P, Abrams J, et al. (2009). Classification of cell death: recommendations of the Nomenclature Committee on Cell Death 2009. Cell Death Differ. 16: 3-11.

Leiter E, Szappanos H, Oberparleiter C, Kaiserer L, et al. (2005). Antifungal protein PAF severely affects the integrity of the plasma membrane of Aspergillus nidulans and induces an apoptosis-like phenotype. Antimicrob. Agents Chemother. 49: 2445-2453.

Majno G and Joris I (1995). Apoptosis, oncosis, and necrosis. An overview of cell death. Am. J. Pathol. 146: 3-15.

McGahon AJ, Martin SJ, Bissonnette RP, Mahboubi A, et al. (1995). The end of the (cell) line: methods for the study of apoptosis in vitro. Methods Cell Biol. 46: 153-185.

Metzstein MM, Stanfield GM and Horvitz HR (1998). Genetics of programmed cell death in C. elegans: past, present and future. Trends Genet. 14: 410-416.

Morris NR (1975). Mitotic mutants of Aspergillus nidulans. Genet. Res. 26: 237-254.

Pontecorvo G, Roper JA, Hemmons LM, Macdonald KD, et al. (1953). The genetics of Aspergillus nidulans. Adv. Genet. 5: 141-238.

Renvoize C, Biola A, Pallardy M and Breard J (1998). Apoptosis: identification of dying cells. Cell Biol. Toxicol. 14: 111-120.

Ribble D, Goldstein NB, Norris DA and Shellman YG (2005). A simple technique for quantifying apoptosis in 96-well plates. BMC Biotechnol. 5: 12.

Rosenberger RF and Kessel M (1967). Synchrony of nuclear replication in individual hyphae of Aspergillus nidulans. $J$. Bacteriol. 94: 1464-1469.

Solary E, Dubrez L and Eymin B (1996). The role of apoptosis in the pathogenesis and treatment of diseases. Eur. Respir. J. 9: 1293-1305.

Statsoft Inc. (2005). Data Abnalysis Software System. Versão 7.1. Available at [www.statsoft.com]. Accessed June 8, 2012.

Stevens JB, Liu G, Bremer SW, Ye KJ, et al. (2007). Mitotic cell death by chromosome fragmentation. Cancer Res. 67: 7686-7694.

Tamm I, Wang Y, Sausville E, Scudiero DA, et al. (1998). IAP-family protein survivin inhibits caspase activity and apoptosis induced by Fas (CD95), Bax, caspases, and anticancer drugs. Cancer Res. 58: 5315-5320.

Telford WG, King LE and Fraker PJ (1992). Comparative evaluation of several DNA binding dyes in the detection of apoptosis-associated chromatin degradation by flow cytometry. Cytometry 13: 137-143.

Tritz R, Mueller BM, Hickey MJ, Lin AH, et al. (2008). siRNA down-regulation of the PATZ1 gene in human glioma cells increases their sensitivity to apoptotic stimuli. Cancer Ther. 6: 865-876.

Wyllie AH (1980). Glucocorticoid-induced thymocyte apoptosis is associated with endogenous endonuclease activation. Nature 284: 555-556. 